\title{
Combined EBL/IBL Nanopatterning on Silicon Nitride Membranes for Time- resolved Magnetic Transmission X-ray Microscopy Experiments
}

\author{
Michal Urbánek ${ }^{1,2}$, Tomáš Šikola ${ }^{1,2}$, Lukáš Hladík ${ }^{2,3}$, Tomáš Hrnčíř ${ }^{3}$ and Jaroslav Jiruše ${ }^{3}$ \\ 1. CEITEC BUT, Brno University of Technology, Brno, Czech Republic \\ 2. Faculty of Mechanical Engineering, Brno University of Technology, Brno, Czech Republic \\ 3. TESCAN a.s., Brno, Czech Republic
}

A study of the magnetic properties of materials and nanostructures is currently one of most attractive areas in solid state physics. Fundamental lengthscales in magnetism, such as exchange length, are in the sub 10-nm range and corresponding fundamental timescales are in the picosecond to femtosecond range. To image magnetization at these length- and timescales can significantly contribute to the understanding of new magnetic phenomena. Magnetic transmission X-ray microscopy (MTXM) is a technique that allows for element specific imaging of magnetic nanostructures at a spatial resolution down to $15 \mathrm{~nm}$ and at a sub-100 ps time resolution [1].

For time-resolved MTXM experiments, samples containing magnetic nanostructures must be carefully designed and fabricated, as they need to allow for transparency of X-rays and must also include waveguides for excitation of the structures by fast magnetic fields. We present nanofabrication of the samples used for studies of the dynamic switching of the spin circulation in tapered magnetic nanodisks [2] at beamline 6.1.2 at the Advanced Light Source (ALS) synchrotron in Berkeley, CA.

The samples were prepared on $2 \times 2 \mathrm{~mm}^{2}, 200 \mathrm{~nm}$ thick silicon nitride membranes. Magnetic field generation was provided by current pulses sent through the 50-nm-thick gold coplanar waveguides, which were pre-patterned on the membranes in the first lithography step (Fig. 1a). We used a Picosecond Pulselabs 10,050A pulse generator capable of pulses from 100 ps to 10 ns with a risetime of $45 \mathrm{ps}$. The output impedance of the pulse generator is $50 \mathrm{ohm}$, the impedance of the coplanar waveguide on the sample was matched to the impedance pulse generator. Because the relative permittivity of the 200-nm-thick silicon nitride membrane $\varepsilon_{\mathrm{r}} \sim 1$, it was necessary to fabricate the grounding plates of the coplanar waveguides in close proximity $(\sim 300 \mathrm{~nm})$ to the central wire to ensure proper impedance. Coplanar waveguides were patterned by electron beam lithography (TESCAN VEGA SEM with DrawBeam lithography software) into a polymethyl methacrylate (PMMA) positive resist mask in a single step by using an extra-large write field of $4 \times 4 \mathrm{~mm}^{2}$. This is possible using Wide Field Optics ${ }^{\mathrm{TM}}$ technology.

In the second lithography step linear arrays of permalloy $\left(\mathrm{Ni}_{80} \mathrm{Fe}_{20}\right)$ nanodisks with diameters in the range of 250-1100 $\mathrm{nm}$ and thicknesses of 20 and $30 \mathrm{~nm}$ were fabricated on the top of the central wire (Fig. 1b). The patterns were written into a 500-nm-thick polymethyl methacrylate (PMMA) positive resist mask by electron beam lithography, and deposition of the permalloy thin film on the pre-patterned mask was done by directional ion beam sputtering with the sputtered particles incident at $15^{\circ}$ from the film normal. Shadowing by the thick PMMA mask led to a wedge-like asymmetry at one side of the disks with a maximum thickness drop of approximately $10 \mathrm{~nm}$. To prevent oxidation, the disks were covered by a 2-nm-thick Au capping layer. The variation of the disk thickness was the same in all disks within the sample. 
In the last step the central wires were trimmed by focused ion beam (TESCAN LYRA instrument) into the desired width $(0.8-2 \mu \mathrm{m})$. It should be noted that we used a FIB-SEM system in which the magnetic field does not penetrate the sample: this is advantageous for magnetic sample applications. The width variation in the wire was used to define amplitude of the magnetic field and by positioning of the line of the disks to the side of the wire it was possible to add out-of-plane magnetic field component (Fig. 1c). The gap to the grounding plates for exact setting of the impedance of the coplanar waveguide was also adjusted within this process [3].

\section{References:}

[1] P Fischer, Materials Science and Engineering R72 (2011) p. 81-95.

[2] V Uhlír et al., Dynamic switching of the spin circulation in tapered magnetic nanodisks, submitted. [3] This work was supported by the project GACR (P102/12/P443), Grant of the Technology Agency of the Czech Republic (TACR) No. TE01020233 and by the European Union FP7 Programme (Contract No. 280566, project UnivSEM).

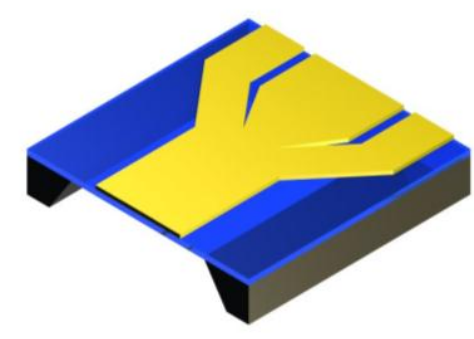

a)

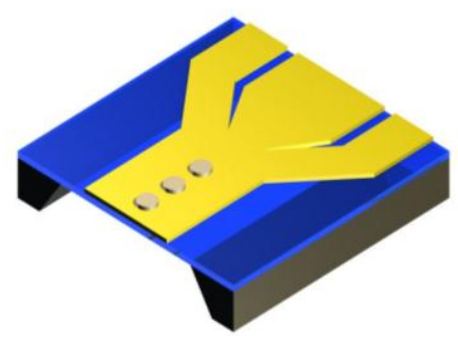

b)

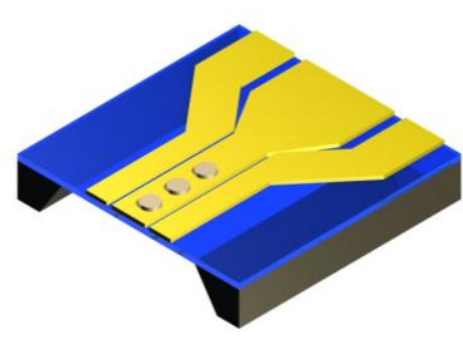

c)

Figure 1. Schematic of the sample - a) A coplanar waveguide fabricated in the first lithography step. b) Magnetic nanostructures fabricated on top of the waveguide in the second lithography step. c) Final trimming of the central wire by FIB. 